\title{
Dispositivo público de telesalud mental durante la pandemia COVID-19: aproximaciones preliminares del Programa Salud Mental Responde (SMR)
}

\author{
Public telemental health during COVID-19 pandemic: preliminary approaches on \\ mental-health help-line program (MHLP)
}

\section{Humberto Lorenzo Persano', María Victoria Kugler², David Alejandro Gutnisky³, Florencia María Alul ${ }^{4}$}

\section{Resumen}

Este trabajo focaliza sobre el desarrollo del programa de atención remota en salud mental dependiente de la DGSAM-Ciudad Autónoma de Buenos Aires durante la pandemia COVID-19. Está fundamentado en los conceptos de telesalud-mental. El cambio llevado a cabo desde un programa previo de orientación telefónica hacia un sistema asistencial con registro en historia integral de salud (HIS 2.0) atendido por profesionales de salud mental, la articulación intersectorial y la posibilidad de ampliación de recursos en redes de cuidados progresivos en salud mental son los aspectos más sobresalientes del dispositivo. También lo es el fortalecimiento de los sistemas de información en salud y la capacitación de profesionales en formación para la atención en telesalud-mental. Durante los primeros seis meses de funcionamiento se atendieron 7.I54 llamados de los cuales el $95,62 \%$ de las situaciones de crisis se resolvieron dentro del protocolo del programa y el $4,38 \%$ restante se derivó para la prosecución de tratamientos por georreferenciación y para re-vinculación con los equipos tratantes respectivos. También fue de utilidad para resolver emergencias psiquiátricas.

El programa resulta novedoso y pionero en nuestro país y resulta ser efectivo para un modelo de atención en salud comunitaria con base en los conceptos de universalidad, gratuidad y accesibilidad. Además, es un trabajo pionero en relevamiento epidemiológico de los usuarios de los sistemas de Salud Mental durante la pandemia COVID-I9.

Palabras clave: Salud mental - Telesalud mental - Telepsiquiatría - Telemedicina - Emergencias psiquiátricas.

\begin{abstract}
This paper focuses on the remote mental health care program developed at DGSAM, in Buenos Aires City during the COVID-I 9 pandemic. It is based on the concepts of telemental health. The change carried out from a previous phone orientation program towards a healthcare system with comprehensive health electronic records (HIS) and carried-out by mental health professionals, intersectorial articulation and progressive mental health care networks are the most outstanding aspects of the program. As well as the strengthening on health information systems and training professionals in telemental health care. During the first six months of operation, 7.154 calls were attended, 95,62\% were solved within the program protocols and the remaining 4,38\% were referred for georeferenced treatments and for re-linking with the respective health care teams. It was also useful in solving psychiatric emergency situations.
\end{abstract}

\footnotetext{
RECIBIDO 15/12/2020 - ACEPTADO 18/1/2021

1. Director General de Salud Mental - Ciudad Autónoma de Buenos Aires (DGSAM)

2. Lic. Psicología (DGSAM)

3. Director de Programas Especiales (DGSAM)

4. Médica Psiquiatra (DGSAM)
} 
The program is innovative and pioneering in our country and turns out to be effective for a community health care model based on the concepts of universality, gratuity, and accessibility. Furthermore, it is the first paper in Argentina on epidemiologic aspects about the mental health system during COVID-19 pandemic.

Keywords: Mental health - Telemental health - Telepsychiatry - Telemedicine - Psychiatric emergencies.

\section{Introducción}

Los antecedentes del programa de atención remota en Salud Mental se remontan al año 1997 y al Hospital "Dr. Braulio Moyano", adonde se brindaba orientación en adicciones. Posteriormente se amplió a todo el campo de la salud mental para, en el año 2000 y en el marco de la Ley $\mathrm{N}^{\circ} 448$ de la Ciudad de Buenos Aires, establecer su sede en la Dirección General de Salud Mental. En septiembre del 2001, el servicio telefónico "Salud Mental Responde", como se denominó, promovía información sobre los recursos de Salud Mental que el Gobierno de la Ciudad de Buenos Aires poseía, y ofrecía contención psicológica a quien lo requería. En el año 2007 se denominó "Servicios Telefónicos - Salud Mental Responde" y "Servicio de Ayuda telefónica en Drogas y Alcohol”. Desde el año 2007 y hasta ahora el programa continuó funcionando ininterrumpidamente en la Dirección General de Salud Mental (en adelante DGSAM) de la Ciudad Autónoma de Buenos Aires. En los últimos años el promedio anual de consultas de dicho programa fue de, aproximadamente, 3.700 Llamados/Consultas. El motivo de consulta de la mayoría de los llamados registrados se debió a una solicitud de información o contención psicosocial telefónica ${ }^{1}$.

Durante la Pandemia por COVID-19, declarada por la OMS el 11 de marzo de 2020 que obligó a decretar un aislamiento social preventivo y obligatorio (ASPO) en la República Argentina a partir del 20 de marzo de 2020, la DGSAM decidió reorganizar dicho programa y actualizarlo debido a las condiciones imperantes en el campo de la asistencia en salud mental en el ámbito de la Ciudad de Buenos Aires. Con el objetivo de proteger a los usuarios del sistema de salud y a los profesionales del riesgo de contagio, favorecer la comunicación con un equipo profesional, extender los días y el horario de atención (oferta) y brindar asistencia por profesionales idóneos en el marco de un contexto imprevisto, inusual, abrupto e incierto como es la pandemia mencionada; en poco tiempo se desarrolló un número telefónico (0800-333-1665) que favore- ció la accesibilidad universal, gratuita y la posibilidad de atención durante las $24 \mathrm{hs}$, todos los días.

Este dispositivo está conformado por un equipo interdisciplinario de profesionales del campo de la salud mental, especialmente médicos psiquiatras y psicólogos y su concepción es un hecho absolutamente novedoso en Argentina, ya que a su vez implica un sistema de derivaciones asistidas con articulación en red entre efectores del campo de la salud mental e interministerial. Sus ventajas no sólo son útiles durante la pandemia COVID-19 sino que lo serán también para los tiempos venideros, tal como acontece en otros países del mundo (Dinakaran et al., 2020; Tsirintani et al., 2020). Puesto que involucra una ampliación de los recursos existentes en salud mental, fortalece los registros en salud y posee una perspectiva de salud integral articulada en redes de cuidados progresivos en salud, tal como fuese planteado por la OMS y la OPS (OPS, 2010), resulta un dispositivo poderoso en la asistencia y organización del sistema de salud mental. Y, como también cumple con el objetivo de fortalecer los sistemas de información en salud de los usuarios de la Ciudad Autónoma de Buenos Aires, a través de la implementación y capacitación en el sistema informático unificado SIGEHOS del Ministerio de Salud de CABA, sirve para planificar estrategias en el abordaje en salud mental a partir de los datos epidemiológicos que surjan de los propios registros de las historias clínicas electrónicas integrales de salud (HIS versión 2.0).

\section{Marco conceptual}

Los primeros registros de telesalud en psiquiatría se remontan a la década del 50 (Shore, 2013) en el Instituto Psiquiátrico de Nebraska (Doyen et al., 2018), mientras que los primeros programas de tele-psiquiatría para niños y adolescentes comenzaron en los años 70 en la Escuela de Medicina del Mount Sinai, ambos en los EE. UU. (Doyen et al., 2018).

A pesar de que en la Ciudad de Buenos Aires el programa de atención telefónica tiene más de dos

1. Fuente DGSAM - Años 2018-2019. 
décadas, el marco conceptual en el cual se decidió su transformación es novedoso debido a que el programa está enmarcado en la asistencia remota en salud mental (Telesalud mental), y en una nueva forma de llevar a cabo prestaciones asistenciales en el campo de la salud mental. Es una primera aproximación de este tipo en el marco de la atención en salud mental comunitaria y muchas veces resulta una oportunidad para acceder al sistema de salud, ser empadronado en el mismo y que la intervención quede registrada en la historia integral de salud (HIS), actualmente en su versión (HIS 2.0). Esta modalidad de registro electrónico en salud fue llevada adelante en la Ciudad de Buenos Aires inicialmente en el primer nivel de atención del sistema público de salud (Faretta et al., 2019). Esta ventaja ya fue registrada por el Sistema de red de portal de telesalud en Carolina del Norte en EE. UU., donde pueden articularse nodos con servicios de emergencia, consulta externa e información electrónica de salud, y donde también pueden realizarse tanto el seguimiento como las consultas y prestaciones realizadas (Saeed, 2018). En Francia la telemedicina se estableció legalmente en el año 2009 bajo el lema: "Hospital, Paciente, Salud, Territorios" poniendo de relieve la relación la salud con las instituciones, el hábitat y el sujeto (Doyen et al., 2018).

La atención remota favorece la accesibilidad a aquellas personas que tienen dificultades, no sólo por las situaciones de la pandemia actual sino por las distancias, los tiempos que insumen los viajes, los costos, las barreras de acceso (Tsirintani et al., 2020) y las situaciones de estigma (Soron, 2016); así como también favorece la articulación con otras áreas de salud y con otros sistemas.

Los cambios tecnológicos actuales han acelerado el crecimiento de esta modalidad asistencial. Aunque el sistema de telesalud es promisorio, quedan aún algunas barreras que resultan en nuevos desafíos para resolver en el futuro inmediato, tales como los aspectos legales y las temáticas relacionadas con la confidencialidad (Hubley et al., 2016). En India han evaluado que la conexión nodal entre primer nivel de atención y el tercer nivel de mayor complejidad, a través de la tele-psiquiatría, también redunda en un beneficio económico en el campo de la salud (Moirangthem et al., 2017).

Por otro lado, se debe entrenar a los jóvenes que están capacitándose en las residencias, concurrencias o postgrados en salud mental en la atención remota en salud bajo estas modalidades para poder afrontar los nuevos desafíos. Promover la accesibilidad, la equidad y la gratuidad cómo principios y valores en su formación académica resulta un logro a desarrollar. La enseñanza de telesalud mental favorecerá a los sujetos que habitan regiones donde el acceso presencial resulta dificultoso y para ello debemos entrenar y capacitar a las nuevas generaciones de especialistas en salud mental y psiquiatría, especialmente para aquellos que desempeñen sus tareas en áreas rurales. Un resumen de los beneficios a ser enseñados es:

a. reducir las disparidades geográficas y económicas en el acceso a los servicios en salud mental;

b. reducir el tiempo de espera y de viaje para el acceso a los servicios de salud mental;

c. mejorar la adherencia terapéutica;

d. mejorar la enseñanza en salud mental;

e. favorecer la coordinación entre los diferentes niveles del sistema de salud mental y

f. aumentar la adherencia de los profesionales a desempeñar la atención en salud mental en las áreas rurales (Saeed et al., 2017).

\section{Material y métodos}

El Programa Salud Mental Responde (SMR) de la DGSAM comenzó a funcionar durante la pandemia COVID-19 por el virus SARS-CoV-2 para dar respuesta a las necesidades de asistencia en salud mental de la población de la Ciudad Autónoma de Buenos Aires, centralizado actualmente a través de una línea telefónica unificada (0800-333-1665) para poder centralizar los llamados y dar respuesta a los mismos mediante un amplio equipo de profesionales durante todos los días y las 24 hs. del día. Esta articulación asistencial en salud mental bajo la modalidad de trabajo remoto en nodos específicos del sistema público de salud y con profesionales también del propio sistema público de salud posibilita una potenciación de los propios recursos en cuidados integrales de la salud. El armado de este dispositivo fue posible gracias al esfuerzo de la propia DGSAM, el apoyo de la Dirección General de Sistemas, la Subsecretaría de Atención Hospitalaria (SSAH) y el Ministerio de Salud del GCABA.

El dispositivo SMR comenzó a funcionar en el mes de abril del año 2020 y para el presente trabajo se utilizaron los registros de los llamados de los primeros seis meses, hasta finales de septiembre del año 2020.

Los registros fueron almacenados en archivos digitales. En el presente trabajo se utilizaron los datos tratados anónimamente y respetando la confidencialidad de los usuarios. Sólo se emplearon dichos registros en forma anónima y se utilizarán para el análisis estadístico descriptivo. El diseño del trabajo es de tipo transversal durante el período mencionado.

La muestra se conformó por un registro longitudinal y aleatorio con un tamaño de 7.154 llamados en 
el período abril-septiembre de 2020. Los datos fueron analizados estadísticamente para obtener un registro de lo que aconteció en el campo de la salud mental en una parte de la comunidad, la que consultó al programa SMR, durante los primeros seis meses de la pandemia COVID-19. Los cuadros clínicos fueron incluidos de acuerdo a la categorización del manual de Clasificación internacional de enfermedades, $100^{a}$ edición (CIE-10) (Organización Panamericana de la Salud, 1995).

\section{Resultados}

El análisis descriptivo de los datos arroja que los llamados fueron realizados mayormente por personas del género femenino (71,9\%), en tanto que los del género masculino correspondió al $(28,1 \%)$ (7.110).

Los llamados provinieron en mayor medida de la comunidad en general, 6.747 llamados (95,3\%), miembros del personal de salud llamaron en 226 oportunidades $(3,2 \%)$ y hubo 4 llamados de personas pertenecientes a poblaciones vulnerables $(0,1 \%)$.

Sobre 6.795 respuestas, 3.572 correspondieron a personas que no poseían cobertura alguna en salud y sólo dependían del sistema público de salud $(52,6 \%)$ y 3.179 de las respuestas $(46,8 \%)$ sí lo poseían, principalmente representado por PAMI (3,2\%), con 214 registros.

La mayor parte de los consultantes que llamaron vivían solos, 2.710 registros (40,6\%); 1.643 registros de llamadas correspondieron a personas que vivían en pareja $(24,6 \%), 1.229$ a personas que viven en familia $(18,4 \%)$ y 637 registros $(9,6 \%)$ a personas que viven con hijos en forma de familia monoparental. La mayoría de los registros de llamados fueron realizados para sí mismo, 5.506 registros $(79,1 \%)$ y 540 registros fueron realizados por problemáticas de sus hijos (7,8\%), 287 registros de llamadas fueron realizados consultando por adultos mayores $(4,1 \%)$ y dos llamados por violencia familiar. Estos datos corresponden a un total de 6.964 registros de llamados.

Con respecto a los antecedentes de llamados previos, la mayoría de estos correspondió a personas que no habían utilizado el sistema previamente, sobre 6.997 registros 4.668 correspondieron a personas que nunca habían utilizado el programa $(66,7 \%)$ mientras que 2.329 sí lo habían utilizado (33,3\%).

Cuando se preguntó acerca de si el llamado correspondía a una situación vinculante al COVID-19, sobre 6.837 registros de llamadas, $6.539(95,6 \%)$ correspondieron a situaciones que no estaban vinculados y $298(4,4 \%)$ registros que sí; desagregados de la siguiente manera $149(2,2 \%)$ personas cursaban la enfermedad por COVID-19, 102 (1,5\%) registros correspondieron a personas recuperadas de COVID-19 y unas 47 personas estaban en estudio ( $0,7 \%)$.

Con respecto al motivo del llamado, sobre un total de 7.060 registros, 3.895 de ellos $(55,2 \%)$ fueron realizados solicitando apoyo psicosocial, 2.263 de los llamados fueron llevados a cabo para solicitar información en el campo de la salud mental. Es importante destacar que 330 registros fueron llevados a cabo $(4,7 \%)$ por preocupaciones acerca de interrupción de tratamientos y acerca de necesidades para retomarlo y 230 registros obedecieron a solicitudes de contención $(3,3 \%)$. Es remarcable que sólo dos personas llamaron para evidenciar que necesitaban medicación psiquiátrica.

Cuando se investigó acerca de tratamientos previos, sobre un total de 6.580 respuestas, 1865 de ellas obedecieron a personas que no tenían antecedentes de tratamientos previos $(28,3 \%)$ y los que respondieron que sí, mayoritariamente habían llevado a cabo tratamientos ambulatorios 4.372 respuestas (66,4\%), 690 de los registros obedecieron a personas que habían recibido alguna internación por motivos de salud mental en algún momento de su vida (10,5\%), 130 de ellos $82 \%$ ) habían recibido tratamiento por salud mental en dispositivos de hospital de día y 41 en comunidades terapéuticas $(0,6 \%)$.

Con respecto a los motivos sintomáticos que promovieron las consultas, sobre 6.731 registros de respuestas, la mayor prevalencia 3.634 (54\%) correspondieron a síntomas de ansiedad/temor. Los trastornos del ánimo ocuparon el segundo lugar con 1.993 $(29,6 \%)$ de los llamados registrados, a su vez la depresión se vio representada específicamente en 17 llamados más (0,3\%). Los síntomas psicóticos ocuparon el tercer lugar con 492 registros (7,3\%). Los síntomas de agresividad/impulsividad ocuparon el cuarto lugar con 315 registros $(4,7 \%)$. El consumo problemático de sustancias se registró en 225 llamados que corresponden al $(3,3 \%)$ del total. Violencia familiar se registró en 132 llamados, correspondientes al (2\%). Los síntomas de angustia se registraron en 48 llamados, representando el $(0,8 \%)$ del total de los llamados. Los síntomas de insomnio se registraron en 24 llamados $(0,4 \%)$ y otros trastornos del sueño a través de otros 9 llamados más, con un $(0,2 \%)$ de casos. Por trastornos del comportamiento alimentario se realizaron 23 llamados, representando un $(0,4 \%)$ de los casos. Los llamados por duelos en el contexto de la pandemia se registraron en 19 casos $(0,3 \%)$ y 2 llamados más por duelos debidos a finalización de relaciones amorosas en el contexto de la pandemia. Los llamados por con- 
flictos familiares representaron 6 de los registros. Los llamados por síntomas hipocondríacos también se registraron en 6 llamados. Los llamados por situaciones de crisis relacionadas con amenazas de suicidio fueron solamente 4 y por falta de medicación psicofarmacológica 1 solo registro.

Durante los primeros seis meses de abordaje desde el programa (SMR) la mayoría de los llamados recibidos fueron resueltos en el marco del propio trabajo de los efectores que recibieron los llamados o durante el seguimiento que el protocolo del programa habilita que corresponde a 3 llamados siguientes. Cuando los casos ameritaron un mayor nivel de seguimiento se procedió a la derivación para la continuidad de los tratamientos o para iniciarlos en zonas georreferenciadas cercanas a su domicilio o para re-vincularizar a los usuarios/pacientes con los respectivos equipos tratantes.

Se realizaron 313 derivaciones a las áreas programáticas de las cuales provenían las demandas (ver tabla 1). De las 313 derivaciones, el 67,4\% (211 casos) se encontraban registradas y evolucionadas en la HIS.2.0SIGHEOS, mientras que el 32,6\% (102) de los usuarios no se encontraban aún empadronados en el sistema.

En cuanto a la cobertura, el 18,5\% (58 casos) poseían obra social o prepaga; mientras que el $81,5 \%$ (255 casos) no contaba con ninguna cobertura.

A su vez, 46,9\% (147 casos) no tomaban ningún psicofármaco al momento de la derivación; mientras que en el 53,1\% (166 casos) sí lo hacía, en algunos casos indicado y en otros automedicado.

Con respecto al 4,38\% de los casos (313) que requirieron derivaciones, la solicitud de estas provinieron de las diferentes áreas programáticas. Las áreas con mayor demanda correspondieron al Hospital "J. M. Ramos Mejía” (54 casos; 17,25\%); Hospital "P. Piñero" (34 casos; 10,86\%) y Hospital "J. A. Fernández” (32 casos; $10,22 \%)$ (ver tabla 1$)$.

La resolución de casos de las 313 derivaciones se distribuyó del siguiente modo: 144 casos iniciaron tratamiento en los diferentes efectores de la salud, 68 casos requirieron reconexión con equipo tratante, 66 casos se debieron reorientar, 10 casos fueron asistidos por el programa ADOP; 4 por el SAME y 21 aún se encuentran en proceso de derivación al momento de este estudio. La gran mayoría de los casos no poseen cobertura de salud $(81,5 \%)$ y pertenecen a la cobertura que brinda el sistema público de salud.

De los casos que requirieron derivaciones, más de la mitad tomaba psicofármacos. Los diagnósticos predominantes, a pesar de ser parecidos en la distribución por frecuencias que, en los casos de resolución sin derivación, los que sí lo requirieron fueron de na-
Tabla I. Distribución de los casos que requirieron derivaciones

\begin{tabular}{lcc}
\hline Área de donde provino la demanda & $\begin{array}{c}\mathrm{n}^{\circ} \text { Casos } \\
(\mathrm{n}=3 \text { I } 3)\end{array}$ & $\%$ \\
\hline HGA “C.Argerich” & 20 & 6.39 \\
\hline HGA “P. Piñero” & 34 & 10.86 \\
\hline HGA “J. M. Ramos Mejía” & 54 & 17.25 \\
\hline HGA “J.A. Fernández” & 32 & 10.22 \\
\hline HGA “T. Álvarez” & 25 & 7.98 \\
\hline HGA “I. Pirovano” & 29 & 9.27 \\
\hline HGA “C. Durand” & 26 & 8.31 \\
\hline HGA “E. Tornú” & 21 & 6.71 \\
\hline HGA “I. Penna” & 25 & 7.98 \\
\hline HGA “A. Zubizarreta” & 7 & 2.24 \\
\hline HGA “F. Santojanni” & 22 & 7.03 \\
\hline HGA “B. Rivadavia” & 5 & 1.60 \\
\hline HGA “D.Vélez Sarsfield” & 13 & 4.15 \\
\hline
\end{tabular}

turaleza más compleja y por ello debieron ser derivados a efectores de salud mental para la prosecución de los tratamientos.

Los cuadros predominantes en los 313 casos que se derivaron a las áreas programáticas de los hospitales de la Ciudad Autónoma de Buenos Aires fueron: Trastorno de Ansiedad (139 casos); Sintomatología Depresiva (81 casos); Esquizofrenia (22 casos); Trastorno Psicótico no especificado (20 casos); Consumo Problemático de sustancias (14 casos); Amenazas de Suicidio (13 casos); Trastorno bipolar (8 casos); Situaciones de violencia (6 casos); TCA (4 casos); Abuso sexual infantil (1 caso) (ver tabla 2).

Tabla 2. Diagnósticos psiquiátricos de los casos derivados a áreas programáticas

\begin{tabular}{lcc}
\hline \multicolumn{1}{c}{ Diagnóstico } & $\begin{array}{c}\text { Cantidad } \\
\text { de casos } \\
(\mathrm{n}=3 \text { I3) }\end{array}$ & $\%$ \\
\hline Trastornos de ansiedad & 139 & 44.43 \\
\hline Síntomas depresivos & 86 & 27.48 \\
\hline Esquizofrenia & 22 & 7.00 \\
\hline Psicosis no especificadas & 20 & 6.40 \\
\hline Consumo de sustancias & 14 & 4.50 \\
\hline Amenazas de suicidio & 13 & 4.16 \\
\hline Trastorno bipolar & 8 & 2.55 \\
\hline Situaciones de violencia & 6 & 1.90 \\
\hline Trastornos alimentarios & 4 & 1.28 \\
\hline Abuso sexual infantil & 1 & 0.30 \\
\hline
\end{tabular}




\section{Discusión y conclusiones}

Los resultados preliminares que surgen del presente estudio evidencian que los sujetos del género femenino consultaron en mayor medida, aunque no es posible determinar si ello obedece a mayor prevalencia de problemáticas o a una mayor apertura a la hora de realizar una consulta en salud mental.

Por otro lado, el personal sanitario utilizó al programa como recurso para abordar las problemáticas que surgieron de la atención a pacientes por enfermedad COVID-19, por lo que el Programa ha brindado apoyo para mejorar el bienestar y, en consecuencia, la salud mental de los propios trabajadores de salud.

Las personas que viven solas demandaron en mayor medida este dispositivo. La mayoría de los llamados fueron hechos por el sujeto que requería la consulta, aunque también se realizaron para vehiculizar consultas para otros familiares: hijos y adultos mayores. Como nuevo dispositivo de atención remota en salud mental no resulta extraño que la mayoría de los llamados se correspondan con personas que nunca lo habían utilizado. Por otro lado, la mayoría de ellos no obedecía a problemáticas relacionadas en forma directa por la infección por el SARS-CoV-2, sino por las consecuencias de la pandemia o por el ASPO. Mayoritariamente se solicitó apoyo psicosocial.

Dos tercios de los consultantes habían tenido algún tipo de tratamiento previo en salud mental, especialmente en forma ambulatoria y sólo un 10,5\% había sido hospitalizado alguna vez. El 54\% de los síntomas corresponden a ansiedad/temor, un $29,9 \%$ a trastornos del ánimo, los síntomas psicóticos (7,3\%), los fenómenos de agresividad/impulsividad (4,7\%) y el consumo problemático de sustancias (3,3\%). La alta prevalencia de síntomas de ansiedad y de trastornos del ánimo se corresponde con un estudio previo realizado en la Argentina, antes de la pandemia, sobre la población general (Cía et al., 2018). En menor medida se observaron trastornos del sueño y problemáticas por desórdenes del comportamiento alimentario. Se detectaron 53 llamados por emergencias psiquiátricas, que se canalizaron y tuvieron resolución adecuada a través del sistema de atención en emergencias SAME.

El programa SMR fue sumamente útil, durante el contexto actual para resolver situaciones de crisis por la sintomatología suscitada durante la pandemia y también por la sintomatología que trajo aparejado tanto el aislamiento social, el distanciamiento emocional, la pérdida de lazos sociales y el distanciamiento afectivo, al cual tuvo que someterse la población por el ASPO.
Cabe señalar que, en nuestro conocimiento, este es el primer trabajo en la Argentina sobre el impacto de la pandemia COVID-19 y el ASPO con datos de los registros de los consultantes en el sistema de salud público, a diferencia de otros trabajos previos sobre el impacto de la pandemia sobre los profesionales (Corral, y Ramírez, 2020) y el ASPO sobre la población general (Observatorio Psicología Social y Aplicada, 2020), que fueron realizados sobre datos obtenidos de encuestas y no sobre prestaciones asistenciales. Esto otorga a este estudio un valor muy específico para el relevamiento epidemiológico en salud mental durante la pandemia por COVID-19, al ser datos obtenidos a partir de registros clínicos.

Como producto de las innumerables dificultades que atravesó la población ante situaciones complejas e inesperadas el programa SMR resultó una herramienta sumamente útil para sobrellevar estas nuevas modalidades de convivencia social y las consecuencias que produjo en la población.

El programa SMR resulta eficaz para resolver situaciones de crisis puesto que el $95,62 \%$ pudo efectivizarse a través de los efectores del mismo y el $4,38 \%$ restante fue derivado para proseguir sus tratamientos en los efectores específicos.

Por otro lado, el programa SMR resultó un dispositivo innovador para organizar la estrategia en el primer nivel de atención en salud mental, favorecer el ingreso al sistema integral de salud público, articular con otros efectores intersectoriales, facilitar la administración de turnos y registrar en la HIS 2.0 los datos de las prestaciones brindadas.

Los resultados de las intervenciones realizadas son alentadores a la hora de afianzar este programa como una modalidad asistencial inicial en salud mental comunitaria y también para la articulación y derivación oportuna entre los distintos niveles de efectores en salud mental.

Conflictos de intereses: los autores declaran no tener conflictos de intereses.

\section{Referencias bibliográficas}

Cía, A. H., Stagnaro, J. C., Aguilar Gaxiola, S. A., Vommaro, H., Loera, G., Medina-Mora, M. E., Kessler, R. C. (2018). Lifetime prevalence and ageof-onset of mental disorders in adults from the Argentinean Study of Mental Health Epidemiology. Social psychiatry and psychiatric epidemiology, 53(4), 341-350.

Corral, R., Ramírez, J. (2020). Impacto de la pandemia COVID-19 en el equipo de salud mental. Rev. Latinoamericana de Psiquiatría, 19(1), 38-42. 
Dinakaran, D., Basavarajappa, C., Manjunatha, N., Kumar, C. N., Math, S. B. (2020). Telemedicine Practice Guidelines and Telepsychiatry Operational Guidelines, India-A Commentary. Indian Journal of Psychological Medicine, https://doi.org/10.1177/0253717620958382

Doyen, C. M., Oreve, M. J., Desailly, E., Goupil, V., Zarca, K., L'Hermitte, Y., Bibay, A. (2018). Telepsychiatry for children and adolescents: a review of the PROMETTED project. Telemedicine and e-Health, 24(1), 3-10.

Faretta, F., Levi, D., Marques, L., Ferrante, D., Bordoni, M. G., Baum, A., de Quirós, F. G. B. (2019). Developing an Electronic Record Tool Representative of Primary Health Care in the Public Health Care System of Buenos Aires City. Stud Health Technol Inform, 264: 516-520. https://doi: 10.3233/ $\underline{\text { SHTI190276 }}$

Hubley, S., Lynch, S. B., Schneck, C., Thomas, M. \& Shore, J. (2016). Review of key telepsychiatry outcomes. World Journal of Psychiatry, 6(2), 269.

Moirangthem, S., Rao, S., Kumar, C. N., Narayana, M., Raviprakash, N., \& Math, S. B. (2017). Telepsychiatry as an economically better model for reaching the unreached: A retrospective report from South India. Indian Journal of Psychological Medicine, 39(3), 271.

Observatorio Psicología Social y Aplicada. (2020). Salud mental en cuarentena: Relevamiento del impacto psicológico a los 7-11 y 50-55 días de cuarentena en la población argentina. Crisis Coronavirus 7. Facultad de Psicología, Universidad de Buenos Aires. https://www.psi.uba.ar/opsa/informes/ Crisis\%20Coronavir
Organización Panamericana de la Salud (2010). Redes Integradas de Servicios de Salud: Conceptos, Opciones de Política y Hoja de Ruta para su Implementación en las Américas. PAHO, Washington DC.

Organización Panamericana de la Salud. (1995). Clasificación Estadística Internacional de Enfermedades y Problemas Relacionados con la Salud, Décima Revisión, Volumen 1, OPS (International Statistical Classification of Diseases and Related Health Problems, 10th Revision 1992).

Saeed, S. A. (2018). Successfully navigating multiple electronic health records when using telepsychiatry: the NC-STeP experience. Psychiatric Services, 69(9), 948-951.

Saeed, S. A., Johnson, T. L., Bagga, M., \& Glass, O. (2017). Training residents in the use of telepsychiatry: review of the literature and a proposed elective. Psychiatric Quarterly, 88(2), 271-283.

Shore, J.H. (2013). Telepsychiatry: videoconferencing in the delivery of psychiatric care. American Journal of Psychiatry, 170(3), 256-262.

Soron, T. R. (2016). Telepsychiatry for depression management in Bangladesh. Int J Ment Health, 45(4), 1-2.

Tsirintani, M., Andrikopoulou, L., Binioris, S. (2020). Contemporary Telemedicine Applications in the Provision of Mental Health Services in Greece. En Androniki, Kavoura, Efstathios, Kefallonitis, Prokopios, Theodoridis (Ed.) Strategic Innovative Marketing and Tourism (pp. 393-401). Springer. https://doi: 10.1007/978-3-030-36126-6_43 